\title{
Biofilm maturity studies indicate sharp debridement opens a time- dependent therapeutic window
}

- Objective: To investigate the hypothesis that newly formed wound biofilms (or bioburdens) are more susceptible to antimicrobial treatment.

- Method: Four separate and distinct models were performed by four separate biofilm research laboratories to evaluate the resistance of biofilms to antimicrobial treatments over time. These included a drip-flow biofilm model along with a hydrodebridement study, a porcine skin punch biopsy ex vivo model, a mouse chronic wound model and clinical longitudinal debridement study.

- Results: All four models showed that, within the first 24 hours, the biofilm community was more susceptible to the selected antibiotics, and after maturing for up to 48 hours became increasingly tolerant. In each model, there was at least a 24-hour period in which the biofilms were more resistant to antibiotics. Each of the models utilised showed a significant decrease in the resistance of the biofilm/ burden to gentamicin for up to 24 hours with a confidence interval of at least $95 \%$. The resistance increased in each of the models by 48 hours and reached original resistance levels by 72 hours.

- Conclusion: These data suggest the principles of biofilm-based wound care, along with the use of serial debridement to continually remove mature biofilm, followed by biofilm wound management strategies, including topical antibiotics while the bioburden is still immature and more susceptible, are valid.

- Conflict of interest: SED is director of Research and Testing Laboratory, a commercial laboratory that develops molecular methods for diagnosis of wounds and infections and CEO of Pathogenius Laboratories, which is a molecular pathogen diagnostic company with a focus on chronic wounds. RDW is medical director of Southwest RegionalWound Care Center and inventor of biofilm-based wound care principles.

In vitro biofilm sensitivity; porcine explants model; mouse surgical excision model; clinical biofilms

R.D.Wolcott,' MD, Medical Director; K.P. Rumbaugh, ${ }^{2} \mathrm{PhD}$, Assistant Professor; G. James, ${ }^{3}$ PhD, Associate Research Professor; G. Schultz, ${ }^{4} \mathrm{PhD}$, Professor; P. Phillips, ${ }^{4} \mathrm{PhD}$, Post-doctoral Associate; Q.Yang, ${ }^{4} \mathrm{MS} \mathrm{Ph}$; C.Watters, ${ }^{2}$ P.S.Stewart, ${ }^{3} \mathrm{PhD}$, Director

S.E. Dowd, ${ }^{5.6} \mathrm{PhD}$, Director;

I Southwest Regional Wound Care Center, Lubbock, TX, US;

2 Texas Tech University Health Sciences Center, Lubbock,TX, US; continued on page 322

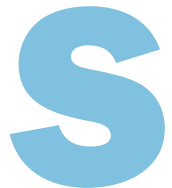

urface-associated bacterial populations (biofilms) are present in chronic wounds, and we propose that biofilm infection is one of the primary barriers to wound healing. Biofilm is removed during debridement, along with necrotic host tissue and other harmful debris, but despite near-universal agreement that debridement is beneficial, there is still controversy as to whether or not sharp debridement improves healing outcomes in chronic wounds. Clinicians largely agree that the removal of devitalised tissue improves wound healing, ${ }^{1-4}$ a concept that is accepted by Center for Medicare Services (CMS) Fiscal Intermediaries, through Local Coverage Decisions. ${ }^{5}$ However, at molecular and cellular levels, there is little evidence to show how debridement exerts a positive effect on wound healing.

Our previous work has demonstrated that bacteria in all of the various stages of biofilm phenotype tightly adhere to the surfaces of chronic wounds. ${ }^{6}$ Although they have been shown to organise around blood vessels and extend into the host in acute wounds, ${ }^{7}$ the majority of chronic wound biofilms seen under scanning electron microscopy and light microscopy appear to reside on the extracellular matrix of the wound bed itself. ${ }^{6}$ Because it accumulates and becomes established quickly, ${ }^{8}$ it is unclear what beneficial effect (if any) removing biofilm may have on wound healing. It is imperative that we better understand the physiology of wound biofilms, especially in terms of their resistance to antibiotics, $^{9,10}$ biocides $^{11,12}$ and host immune mediators ${ }^{13,14}$ during biofilm maturation.

Biofilms are well documented, both generally and in their developmental stages. ${ }^{15-19}$ In short, planktonic (single cell, non-attached) bacteria undergo a phenotypic metamorphosis through attachment, micro-colony formation (where they are metabolically more active) and then progress into mature biofilms with a protective matrix, which can be derived from a wide variety of host-supplied, environmentally derived and bacterially produced building blocks. ${ }^{20}$ Genotypic and phenotypic heterogenicity is a seminal survival strategy for mature biofilms. ${ }^{21-23}$

Therefore, it follows that if a biofilm's resistance increases as it matures and its metabolic rate declines, 
3 Center for Biofilm Engineering, Montana State University, Bozeman MT, US;

4 Institute for Wound Research, University of Florida, Gainesville, FL, US;

5 Research and Testing Laboratory, Lubbock, TX US;

6 Medical Biofilm Research Institute, Lubbock, TX, US. Email: sdowd@ pathogenresearch.org any procedure that physically disrupts the biofilm, such as sharp debridement, may create a 'window of opportunity' by removing the bulk of mature biofilm. The metabolic rate of residual microorganisms is forced to increase as biofilm grows back. ${ }^{8,13,24-28} \mathrm{Cli}$ nicians might take advantage of this short time between debridement and biofilm re-accumulation and maturation, as a therapeutic window.

This study aimed to determine if these timedependent and tightly-integrated biofilm defences can be exploited. We sought to examine the properties of early reforming biofilm ( 24 hours old), which has a less organised structure, a more active metabolism and a less pronounced stress response, ${ }^{8,13,24-28}$ compared with older, more mature biofilms. Specifically, we wanted to test if the 'young biofilm' phenotype is more susceptible to treatment agents, including antibiotics, than mature biofilms.

\section{Method}

\section{Bacteria and culture conditions}

Staphylococcus aureus strain ATCC \#29213 was obtained from the American Type Culture Collection. Pseudomonas aeruginosa strain mPAO1 was obtained from the University of Washington Genome Center (www.genome.washington.edu/ UWGC). (Reference strains were selected to allow reproducibility for future studies.) Frozen stock cultures were maintained at $-70^{\circ} \mathrm{C}$. Overnight cultures were initially propagated from frozen stocks in 100\% Tryptic Soy Broth (TSB, Becton, Dickinson and Company, BD, Sparks, MD) at $37^{\circ} \mathrm{C}$.

\section{Drip-flow biofilm model}

In vitro biofilm susceptibility testing was conducted using $P$. aeruginosa and $S$. aureus biofilms grown in drip flow reactors (DFRs, Biosurface Technologies Corporation, Bozeman, MT) similarly to previously described experiments. ${ }^{29}$ The DFR method also forms the basis of ASTM Standard Test Method E 2647-08 for the growth of $P$. aeruginosa biofilms (ASTM International). Briefly, DFRs were equipped with hydroxyapatite-coated glass slides (Clarkson Chromatography, South Williamsport, PA) and operated at room temperature in line with the ASTM protocol. Sterile DFRs were conditioned with sterile medium for 20 minutes and inoculated with $1 \mathrm{ml}$ of an overnight culture of $P$. aeruginosa or $S$. aureus. The growth medium consisted of 1\%-strength brain-heart infusion broth (BD) supplemented with $0.5 \%$ adult bovine serum (HyClone, Logan, UT). After inoculation, the DFR was placed in a horizontal position for two hours, to allow bacterial attachment to the slides. Subsequently, the device was placed at a $10^{\circ}$ angle, with sterile medium dripping on the slides at a rate of $10 \mathrm{ml} / \mathrm{h}$ on each slide.

After the predetermined biofilm growth time (range 6-12 hours), the slides were removed and placed into tubes containing $10 \mathrm{ml}$ of saline solution (control) or $10 \mathrm{ml}$ of saline solution containing $200 \mu \mathrm{g} / \mathrm{ml}$ gentamicin (Sigma, Milwaukee, WI) for 24 hours. After the treatment period, the biofilms were rinsed and scraped from the slides into $10 \mathrm{ml}$ of Dey-Engley Neutralization broth (BD) and then vortexed (30 seconds), sonicated (two minutes), and vortexed (30 seconds) again, to create a biofilm suspension. This suspension was then serially diluted with phosphate buffered saline (PBS), plated on tryptic soy broth (BD) and the plates were incubated at $37^{\circ} \mathrm{C}$ for $24-48$ hours. Colony-forming units (CFUs) were then counted and the biofilm viable cell density was calculated based on the dilution plated and the surface area of the slide. Biofilm densities were logarithmically transformed and the mean log density was calculated from at least three repeat experiments.

\section{Biofilm regrowth after hydrodebridement}

Biofilms were grown for 48 hours, as described above, and were then placed in a holder and sprayed for approximately 20 seconds with saline (approximately $150 \mathrm{ml}$ of fluid) using a Xomed Hydrodebrider System (Medtronic Inc., Jacksonville, FL). The spraying was done with both side-to-side and upand-down sweeping motions so that all areas were sprayed twice, once in each axis. ${ }^{29}$ Following this 'debridement', slides were either immediately treated with saline and gentamicin as described above, or returned to the DFR for additional biofilm growth (regrowth). Following the predetermined regrowth period, the remaining slides were treated with saline and gentamicin as described above.

\section{Biofilm on porcine explants model}

Because porcine skin has been shown to have the greatest similarity to human $\operatorname{skin}^{30}$ and is readily available, it was chosen as the model substratum for biofilm growth on skin.

The key parameters of this model include preparing chlorine gas-sterilised fresh porcine skin explants ( $8 \mathrm{~mm}$ diameter and $3-4 \mathrm{~mm}$ thick), mechanically creating partial-thickness 'wound beds' ( $2 \mathrm{~mm}$ diameter and $1.5 \mathrm{~mm}$ deep), and inoculating them with early logarithmic phase bacterial suspension culture. The biofilm was then cultured on the explant ( $\mathrm{n}=4$ for each data point), which was placed on a tryptic soy $0.5 \%$ agar (soft TSA) medium to maintain the moisture required for bacterial growth, with the addition of an appropriate antibiotic to limit bacterial growth to the explant 'wound bed', with the intention of inhibiting penetration of the biofilm through to the bottom of the explant. Soft TSA containing $50 \mu \mathrm{g} / \mathrm{ml}$ gentamicin for PAO1 and $250 \mu \mathrm{g} / \mathrm{ml}$ oxacillin for SA35556 was used. Explants were transferred to fresh antibiotic agar plates daily. 
The biofilms were cultured on sterile explants for 1-5 days. Biofilm explants were then processed with or without an additional 24-hour treatment in TSB containing the appropriate antibiotic to kill susceptible planktonic bacteria $(200 \mu \mathrm{g} / \mathrm{ml}$ gentamicin for PAO1 or $1 \mathrm{mg} / \mathrm{ml}$ oxacillin for SA35556). The explants were sonicated in $7 \mathrm{ml}$ PBS with $5 \mathrm{ppm}$ Tween 80 . The resulting bacterial suspensions were serially diluted and each dilution was plated in triplicate on TSA plates. Bacterial levels are expressed as the average colony forming units per $\mathrm{ml}(\mathrm{CFU} / \mathrm{ml})$ found in the bacterial suspension.

\section{Mouse surgical excision wound model}

Our model is based on the chronic wound model described by Brown and Greenhalgh, ${ }^{31}$ and generates a full-thickness, slow-healing wound that remains open and chronically infected with high levels of $P$. aeruginosa for up to 3 weeks.

Wild-type Swiss Webster mice were anesthetised, shaved and administered a dorsal, full-thickness 1.5 $\mathrm{x} 1.5 \mathrm{~cm}$ surgical wound as previously described. ${ }^{31,32}$ A total of 10E4 $P$. aeruginosa were applied to the surface of these wounds. These were covered with a sterile gauze pad, which were held in position with adhesive, transparent, semipermeable polyurethane dressings (OpSite, Smith \& Nephew). This dose of $P$. aeruginosa has been empirically determined to cause an effective chronic wound infection in mice. ${ }^{31}$ (Brown 1997) We used a common laboratory, fully virulent strain of $P$. aeruginosa (PAO1). ${ }^{33}$

At one, two and three days postoperatively ( $n=9$ per time point), the mice were sacrificed and the gauze pads removed from their wounds. The gauze pads, which were covered with thick wound exudate, were cut into thirds and placed in sterile saline, $100 \%$ bleach for 20 minutes, or a $200 \mu \mathrm{g} / \mathrm{ml}$ gentamicin solution for 5 hours. Gauze sections were then removed from the bleach or gentamicin solution and neutralised for 10 minutes in 10\% sodium L-ascorbate or Dey-Engley Neutralizing broth, respectively. All gauze sections were then placed into sterile saline and vigorously sonicated and vortexed. The resulting solution was serially diluted and plated on Luria-Bertani (LB) agar to determine the CFU/g of bandage. To determine the percentage of cells viable after antimicrobial treatment, the CFU/g after treatment was divided by the CFU/g from the untreated section of gauze and multiplied by 100 .

\section{Clinical biofilms}

Three patients were identified with large $\left(>10 \mathrm{~cm}^{2}\right)$ non-healing venous leg ulcers (VLUs), diagnosed as having high proportions of $P$. aeruginosa in their wound biofilms (Pathogenius diagnostics, Lubbock, TX). These patients gave consent for the analysis of material removed from their wounds, utilising standard sharp debridement methods (Western
Institutional Review Board approved protocols 56-RW-004 WIRB Protocol \#20062347). Seven days before the study, all the wounds were debrided using standard sharp debridement techniques. The wounds were treated with standard care saline 'wetto-dry' dressings every 12 hours for 3 days before the study. On day 0 , the mature wound biofilm was removed from the entire wound bed by sharp debridement and sent for analysis. Each wound was then divided into three zones. On day 1, zone one was debrided utilising a curette and the material removed was placed into sterile Eppendorf tubes and sent immediately for analysis. Samples were processed within 30 minutes of collection \pm 15 minutes. This process was repeated for zone 2 on day 2 and zone 3 on day 3 .

On receipt in the laboratory, biofilm debridements were removed and placed into tubes containing $10 \mathrm{ml}$ of saline solution (control) or $10 \mathrm{ml}$ of saline solution containing $200 \mu \mathrm{g} / \mathrm{ml}$ gentamicin (Sigma) for 24 hours. After the treatment period, the biofilms were centrifuged, washed twice and placed in $10 \mathrm{ml}$ of Dey-Engley Neutralising broth and then vortexed (30 seconds), sonicated (two minutes), and vortexed (30 seconds) three times, to create biofilm suspensions. These were then serially diluted with PBS, plated on TSB and the plates were incubated at $37^{\circ} \mathrm{C}$ for $24-48$ hours. CFU were then counted and the biofilm viable cell density was calculated based on the dilution plated and the surface area of the specimen. CFUs plotted on a log scale were calculated from at least three technical repetitions (separate plates counts) for each point.

\section{Statistical analysis}

Clinical biofilm data were analysed using NCSS 2007 (Kaysville, UT) AND XLstat (Addinsoft, New York, NY). Mouse surgical model data were analysed using InStat (La Jolla, CA). Other statistics were run with SPSS and Excel.

\section{Results \\ Drip-flow biofilm model}

$P$. aeruginosa biofilms grown for 6 and 12 hours were susceptible to treatment with gentamicin, whereas after 24 and 48 hours of growth, they became highly tolerant to gentamicin treatment (Fig 1). Log reductions, based on the differences between the control (saline) and gentamicin treatments in each experiment, had means ( \pm standard errors) of $2.5 \pm$ 0.4 and $3.0 \pm 0.9$ for six and 12-hour biofilms, respectively (two-tailed Kruskal-Wallis test, $\mathrm{p}<0.05$ ). After 18 hours of biofilm growth, gentamicin was less effective (Fig 1) with a mean log reduction of 1.4 \pm 0.8 . No differences were detected in CFU counts between control biofilms and gentamicin-treated biofilms once the biofilms had matured for 24 and 48 hours prior to treatment. 
Fig I. Log ( 10$)$ Pseudomonas aeruginosa biofilm density versus biofilm growth time

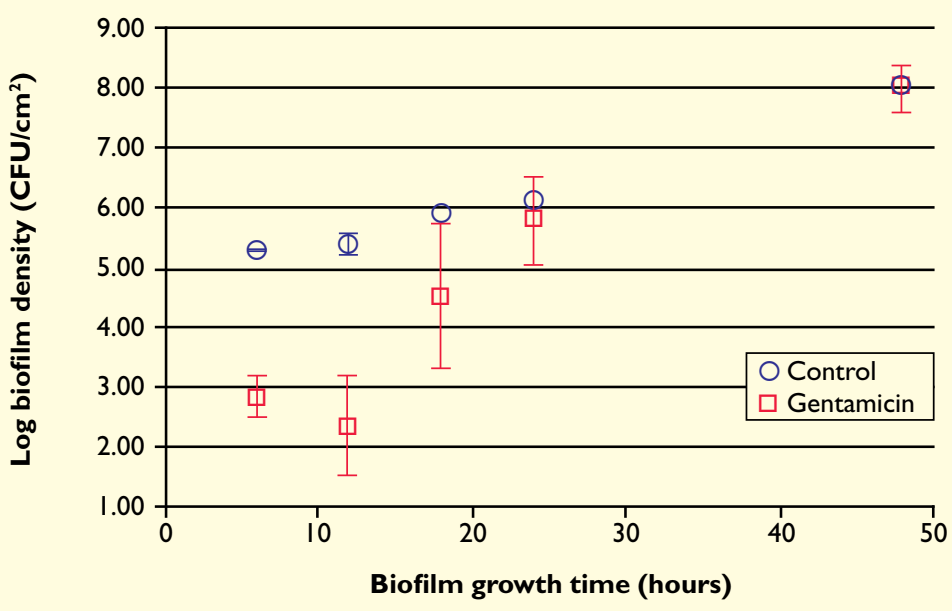

After growth for the designated amount of time, the biofilms were treated with saline (control) or saline containing $200 \mu \mathrm{g}$ of gentamicin for 24 hours. The biofilms were relatively susceptible to the antibiotic after 6-12 hours of growth but then became more tolerant and were completely tolerant after 48 hours of growth

The error bars show \pm one standard deviation of the means

Fig 2. Log (10) Staphylococcus aureus biofilm density versus biofilm growth time

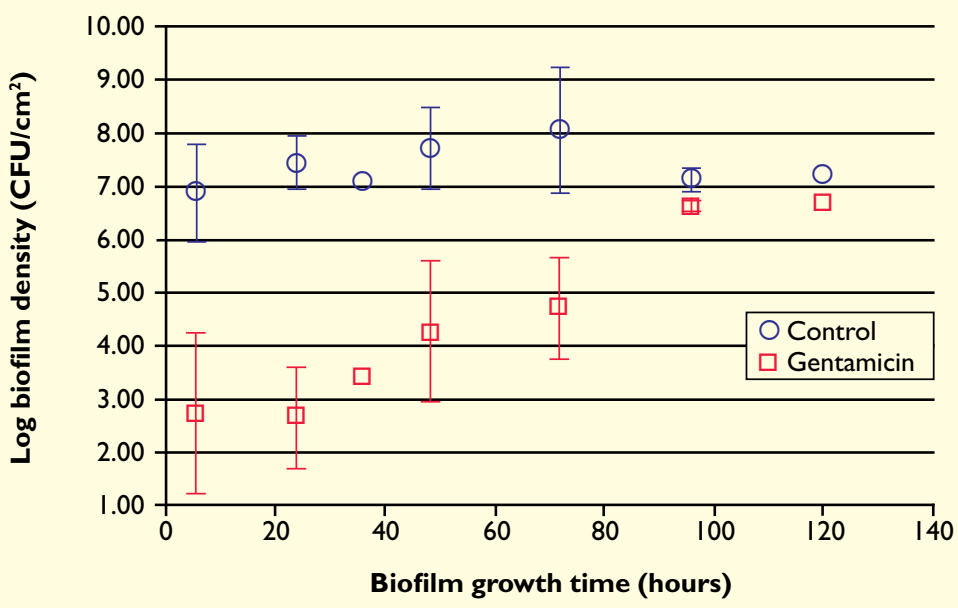

After growth for the designated amount of time, the biofilms were treated with saline (control) or saline containing $200 \mu \mathrm{g}$ of gentamicin for 24 hours. The biofilms were relatively susceptible to the antibiotic after 6-72 hours of growth but became more tolerant and were completely tolerant after 96 hours of growth

The error bars show \pm one standard deviation of the means

The $S$. aureus control biofilms rapidly reached considerably higher cell density than the $P$. aeruginosa control biofilms under identical conditions; for example, after 6 hours the mean log biofilm density was $6.9 \pm 0.9$ for $S$. aureus and only $5.3 \pm 0.2$ for $P$. aeruginosa. However, the log cell densities of the control biofilms formed by these two species were equivalent after 48 hours ( $7.7 \pm 0.8$ and $8.0 \pm 0.5$ for $S$. aureus and $P$. aeruginosa, respectively).

Regardless of reaching higher biofilm densities more quickly, the $S$. aureus biofilms took longer to achieve tolerance to gentamicin (Fig 2). Although tolerance increased as $S$. aureus biofilms matured over the first 72 hours, a mean log reduction was $3.3 \pm 1.4$ was still achieved after 72 hours. By 96 hours the $S$. aureus biofilms were completely tolerant, with no significant differences detected between CFU counts of control biofilms and gentamicintreated biofilms.

\section{Biofilm regrowth after hydrodebridement}

Treatment with the Hydrodebrider and saline of $P$. aeruginosa biofilms grown for 48 hours resulted in biofilm log densities ranging from 3.1 to 4.4 . In contrast, the log density of biofilms grown for 48 hours and treated with static saline were 7.3 to 8.3. Thus, the hydrodebidement resulted in an approximate 4 log reduction in viable biofilm bacteria.

The mature biofilm remaining after debridement was tolerant to gentamicin treatment and only slightly susceptible after 6-24 hours of regrowth (Fig 3 ). The six-hour regrown biofilms (Fig 3) were much more tolerant to gentamicin than the biofilms grown for six hours (Fig 1), with a mean log reduction of $1.1 \pm 0.3$ for a six-hour regrown biofilm compared with $2.5 \pm 0.4$ for a nascent six-hour biofilm.

Treatment with the Hydrodebrider and saline of $S$. aureus biofilms grown for 48 hours resulted in biofilm log densities ranging from 1.3 to 3.2. The log density of biofilms grown for 48 hours and treated with static saline were 2.1 to 4.9 . Thus, the hydrodebidement resulted in a 1 to 3 log reduction in viable biofilm bacteria (Fig 4).

The biofilm remaining after debridement and regrowth had a similar gentamicin tolerance to nascent 48-hour $S$. aureus biofilms. For nascent 48-hour $S$. aureus biofilms, the mean log reduction was $3.2 \pm$ 1.3 (Fig 2); the mean log reduction of debrided 48-hour biofilms was $2.5 \pm 0.7$, whereas the $\log$ reduction was $2.4 \pm 0.2$ after 24 hours of regrowth. After 24 hours of regrowth there was only a slight reduction in the gentamicin-treated biofilm relative to the control (log reduction 0.88) and the biofilms were completely tolerant to gentamicin after 72 hours of regrowth.

\section{Biofilm on porcine explants model}

The results demonstrate that the early (24-hour) planktonically seeded explants were significantly susceptible to the appropriate antibiotic for both $P$. aeruginosa and $S$. aureus. Antibiotic tolerance to 100 $\mathrm{x}$ minimum inhibitory concentration (MIC) antibi- 
otic treatment approached maximal levels after 48 hours, and reached a tolerance plateau after 3 days. The growth curves of biofilm bacteria (24-hour antibiotic-treated explants) were compared with untreated explants in order to assess the presence and growth pattern of 'functionally mature' bacterial biofilms. The presence of bacterial biofilm was visually verified by light and scanning electron microscopy (data not shown). P. aeruginosa PAO1 and $S$. aureus SA35556 formed fully mature biofilm on $8 \mathrm{~mm}$ porcine explants after 3 days. While $S$. aureus SA35556 consistently showed a delay in biofilm formation compared with PAO1, the SA35556 biofilm penetrated through the bottom of the porcine explants after 4 days and could not be restricted to the surface of the explants during 24-hour treatment in liquid media containing $1 \mathrm{mg} / \mathrm{ml}$ oxacillin. Consequently, data points were not plotted for day 4 and 5 on the SA35556 growth curves in Fig 5.

\section{Mouse surgical excision wound model}

The percentage of $P$. aeruginosa PAO1 viable after bleach and gentamicin treatments increased with the age of the wound (Fig 6). Dramatically, we saw that at postoperative day 1 only $0.01 \%$ of PAO 1 were tolerant to bleach treatment, but by day 4 over $39 \%$ were still viable after being treated with $100 \%$ bleach.

The bacteria in the wounds also became increasing tolerant to gentamicin, increasing from $0.23 \%$ of cells in one-day-old wounds to $8.33 \%$ in two-dayold wounds still viable after gentamicin treatment. Tolerance to gentamicin plateaued after postoperative day 2 . These data indicate that the $P$. aeruginosa in these chronic wounds develop resistance to antimicrobials over just a few days, which suggests they are biofilm-associated.

\section{Clinical debridement}

One week after debridement the patients' bioburden was removed via sharp debridement and the sample was evaluated for the ability of gentamicin to kill bacteria within the biofilm. Fig 7 shows that there was an average $4 \log$ reduction in bacteria after antibiotic treatment of the mature (prestudy) biofilm. At baseline, the three VLUs chosen for the study all had significant and high levels of $P$. aeruginosa (average $5.2 \times 10^{8} \mathrm{CFU} / 5 \mathrm{mg}$ bioburden). After initial debridement the bacterial populations rapidly grew back to the original concentrations. However, at 24 hours post-debridement all of the biofilms were more susceptible to antibiotic treatment showing greater than a nine-fold reduction in concentration in relation to the control biofilm and a significant difference was observed between the susceptibility of day 0 predebridement and day 1 (24 hours) post-debride-
Fig 3. Log ( 10$)$ biofilm density for debrided (0 hours) biofilms of Pseudomonas aeruginosa and biofilms that were debrided and then regrown for the specified amount of time

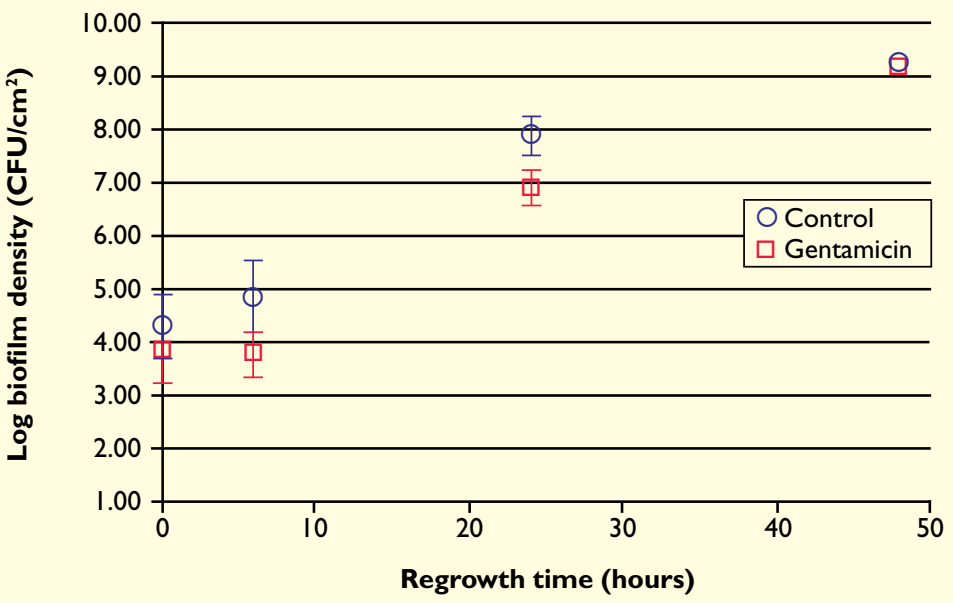

The biofilms were grown for 48 hours and then treated with saline (control) or saline containing $200 \mu \mathrm{g}$ of gentamicin for 24 hours immediately after debridement or after debridement and regrowth. P. aeruginosa biofilms were only slightly susceptible to the antibiotic after debridement and regrowth

The error bars show \pm one standard deviation of the means

Fig 4. $\log (10)$ biofilm density for debrided (0 hours) biofilms of Staphylococcus aureus and biofilms that were debrided and then regrown for the specified amount of time

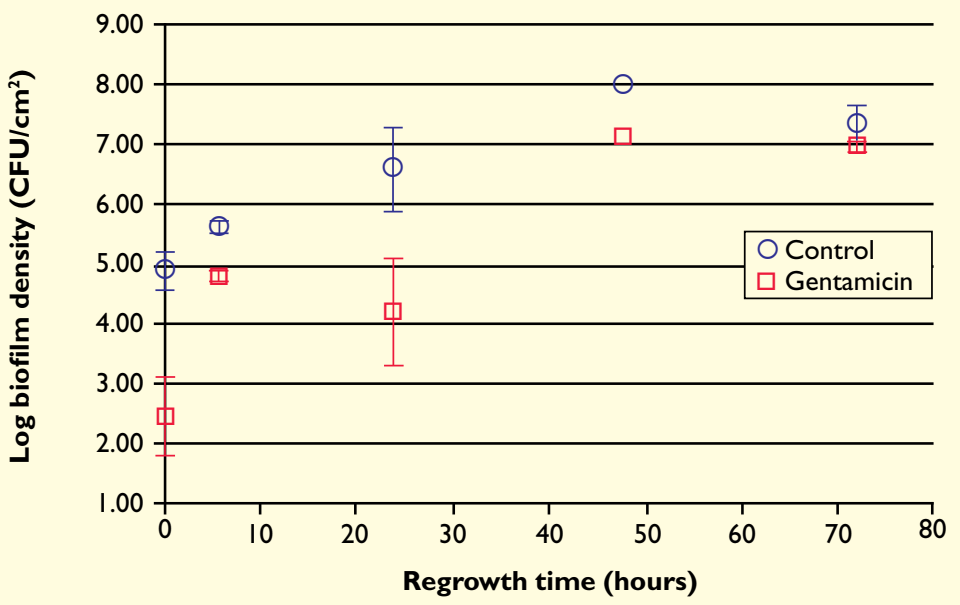

The biofilms were grown for 48 hours, then debrided and treated with saline (control) or saline containing $200 \mu \mathrm{g}$ of gentamicin for 24 hours immediately after debridement or after debridement and regrowth. S. aureus biofilms were tolerant to antibiotic treatment after debridement and regrowth

The error bars show \pm one standard deviation of the means.

ment (two-tailed Kruskal-Wallis test, $\mathrm{p}=0.05$ twotailed T-test, $\mathrm{p}<0.001)$. At 48 hours two of the debridements still showed higher sensitivity to 
Fig 5. Growth curves of bacterial biofilm cultured on porcine skin explants.

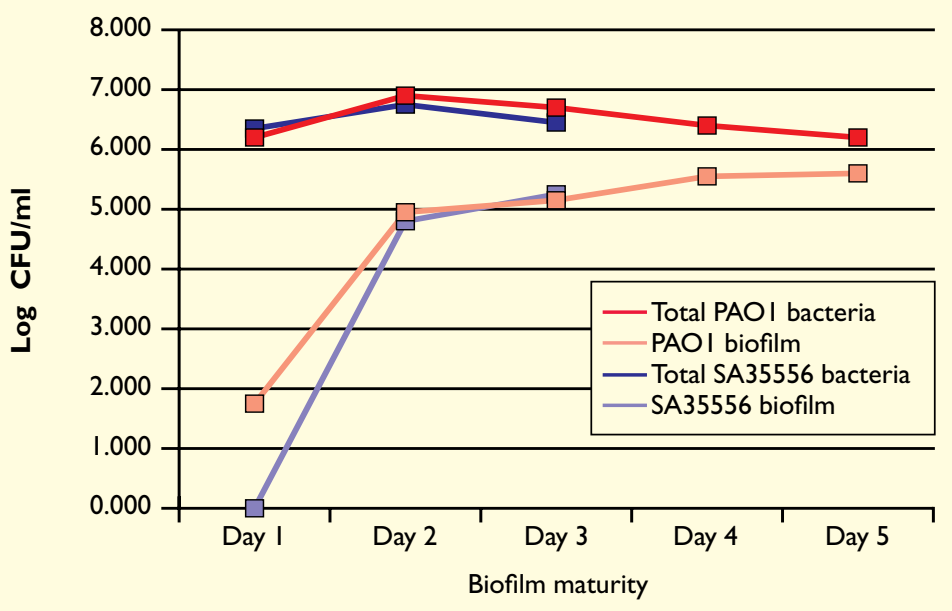

The growth curve shows the log CFU/ml found on explants without antibiotic treatment (total bacteria) or following 24-hour incubation of the explants in $200 \mu \mathrm{g} / \mathrm{ml}$ gentamicin (PAOI) or $\mathrm{Img} / \mathrm{ml}$ oxacillin (SA35556) to kill planktonic bacteria (antibiotic-tolerant biofilm). Bacterial levels at each condition are expressed as the log of the CFU/ml (average) found in the bacterial suspension of the explants sonicated in $7 \mathrm{ml} \mathrm{PBS} / 5$ ppmTween 80

The error bars \pm indicate the standard deviation

Fig 6.The resistance of Pseudomonas aeruginosa in murine surgical excision wounds to antimicrobials increases over time

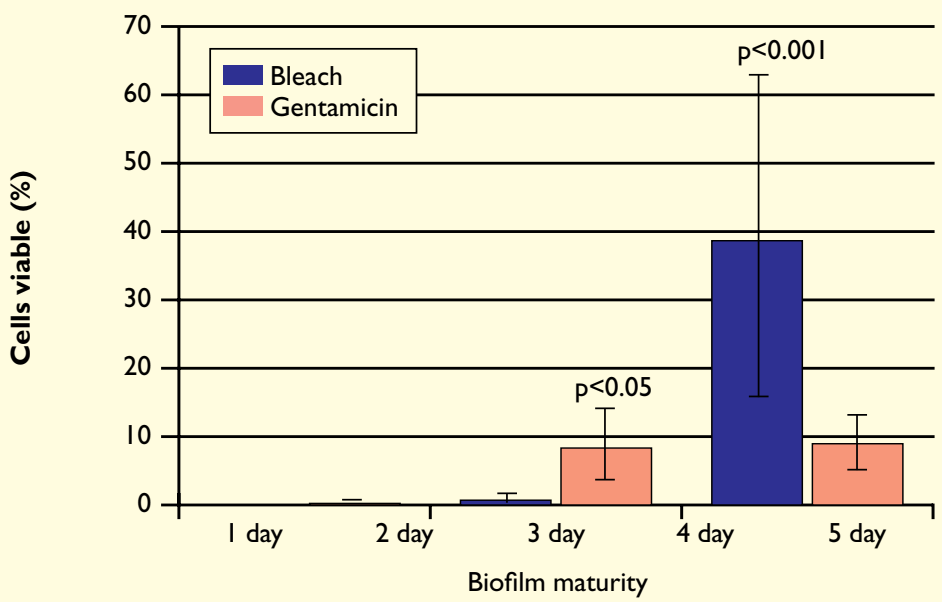

Murine surgical wounds were infected with PAOI and covered with a sterile gauze pad. At postoperative days I, 2 or 4, the gauze was removed and the tolerance of the bacteria to either bleach or gentamicin was determined as described in the text. Shown are the percentage of PAOI cells that were still viable after bleach or gentamicin treatment $(n=9$ mice/time point, KruskalWallis test $p<0.05$, Fisher-exact $(p<0.05)$

antibiotics, while one of the bioburden samples had regained resistance $(\mathrm{p}>0.05$, when compared with day 0 ). At 72 hours the biofilms were back to nearly the same susceptibility levels as original mature biofilm.

\section{Discussion}

The in vitro experiments reported here-demonstrate that bacterial biofilms develop antibiotic tolerance over time with less mature biofilms being more susceptible to antibiotic treatment. This occurs relatively quickly with $P$. aeruginosa biofilms (24 hours) and more slowly with $S$. aureus biofilms (96 hours). Partial removal of the biofilm in in vitro models did not increase tolerance in the remaining biofilm.

Previous research with both $P$. aeruginos $a^{26}$ and $S$. epidermidis ${ }^{27}$ has shown that bacteria in the deeper layers of biofilm were inactive relative to those nearer the air interface. Thus, removal of the active cells from the surface of the biofilm may expose dormant bacteria that have reduced susceptibility to antibiotic treatment. Our results suggest that complete removal is necessary to prevent the persistence and regrowth of antibiotic-tolerant biofilms and only newly formed biofilms are susceptible to antibiotic treatment. In actual chronic wounds a sharp debridement study was utilised to validate these findings, confirming that following removal of the mature biofilm the biofilm presented with a 24-48 hour therapeutic window.

Wound biofilm, or biofilm adapted to the host wound niche, exhibits genotypic and phenotypic diversity. ${ }^{34}$ This diversity is directly responsible for multiple biofilm defences against antibiotics such as impeded penetration, decreased growth/metabolic rate, stress response and modulation of quorum sensing pathways. ${ }^{8,13,24-28}$ It is the simultaneous expression of these defences in conjunction with the biofilm structure, and combined with the incalculable synergies between multiple microbial species, that can prevent the eradication of polymicrobial infections ${ }^{21}$ caused by biofilm.

Impaired penetration caused by the biofilm selfsecreted matrix may be much more important relative to biocides than to antibiotics. ${ }^{9,10,35-38}$ Biocides, by nature, tend to be reactive (ie, are usually charged molecules with reactive molecular motifs) and thus are consumed by the extracellular polymeric substances of the biofilm. Although antibiotics can react, they can be adsorbed, limited by molecular weight or electrostatically influenced by the biofilm matrix..$^{10}$ It is clear that most antibiotics are not perturbed significantly by the glycocalyx (extracellular polymeric material [glycoprotein] produced by some bacteria)..$^{38-40}$

Another bacterial defence provided by the biofilm is the slow growth/metabolic rate, which protects against antibiotics that target highly metabolic bacteria. Indeed, some of the most effective antibiotics target metabolic machinery or cell wall synthesis 
directly relating to cell metabolism or growth. Bacteria associated with immature biofilms, however, are postulated to be highly active as the bacteria engage in reaching a climax community and, ultimately, a mature stable biofilm. Unless biofilms in chronic wounds are continually forced to remain in an immature state, where they are more metabolically active, such as during reformation after debridement, they have the potential to be hundreds of times more resistant than their planktonic phenotype. ${ }^{10,39}$

Indeed, the data from our in vivo mouse wound biofilm and clinical biofilm maturity experiments also suggest that early biofilm re-accumulation in the host niche correlates with the pattern seen in our in vitro models. There was increased sensitivity to the antimicrobials tested in one-day-old biofilms as compared with mature, four-day-old biofilms. Thus, this increased sensitivity seen in newly reforming biofilms may be therapeutically useful. In all the models we can deduce that, after 48 hours, the biofilm can reach maturity and be very resistant to antibiotic therapy, even with higher doses applied topically. We now also have some clinical evidence that effective debridement initiates a limited window of therapeutic opportunity when antimicrobials may be more effective. By taking advantage of this window of opportunity we can extend the time before the biofilm again reaches maturity and target bacteria when they are more susceptible (highly metabolic and without mature biofilm matrix protection).

Sharp debridement has become the most utilised and important tool in managing chronic wounds around the world. Yet very little is known as to why (or even if) debridement improves chronic wound healing. This study raises the possibility that sharp debridement forces wound biofilm to reconstitute itself (regrow) and the resulting 'young' biofilms (or the remaining adhered or newly adhered bacterial cells) are more susceptible to antimicrobial and antibiofilm agents. We feel this property of wound biofilms opens an exciting new area of research to define the molecular and cellular roles involved in impaired wound healing and, more importantly, targeted treatments.

This, in turn, gives the host a window of opportunity where it is not actively fighting a biofilm infection and can recruit its resources to promote healing. This supports the role of serial debridement as part of biofilm-based wound care as a primary tool to keep biofilm from reaching maturity and extending the therapeutic and healing windows of opportunity.

Each of the current studies relies on relatively small numbers of replications $(n=3$ or $n=4)$ for each data point. However, the four individual studies, which were all performed by independent medical
Fig 7.The results of clinical biofilm treatment with antibiotics

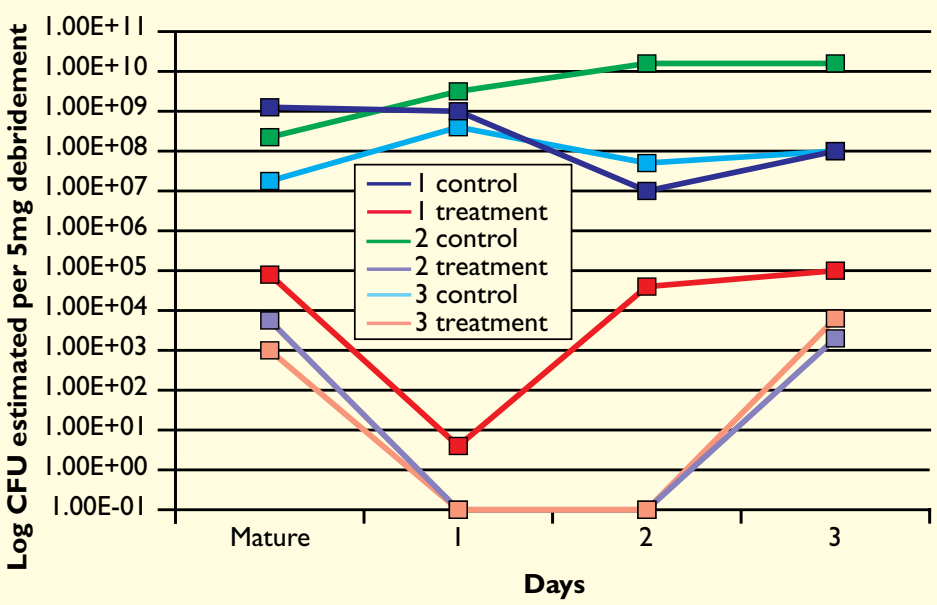

Fully mature biofilms from patients were evaluated for their susceptibility to gentamicin. There are three control samples and three gentamicin treatment paired-samples from three patients with venous leg ulcers. The results indicate that mature biofilm is highly tolerant to treatment with high doses $(200 \mu \mathrm{g} / \mathrm{ml})$ of gentamycin and that within 72 hours the biofilm reconstitutes its resistant phenotype. Graph indicates average CFU for each time point and each sample is plotted on a log scale. There is no significant difference in controls over time. In the treatment the mature or day 0 pre-debridement is significantly more resistant to the antibiotic than the day I sample $(p=0.05)$

biofilm research laboratories, all reached the same conclusion. In our opinion, this strengthens the overall power of the results and generalisability of the conclusions.

\section{Conclusion}

The results of the first in vitro model showed that after growth and treatment with either saline (control) or saline containing $200 \mu \mathrm{g} / \mathrm{ml}$ of gentamicin (treatment) the biofilms were relatively susceptible to the antibiotic at both 6 and 12 hours but after this became increasingly tolerant and then apparently reached a plateau for tolerance after 48 hours. The results of the second in vitro study demonstrate that a planktonic seeded mammalian matrix (porcine explant) is susceptible to antibiotics during the first 48 hours of development into biofilm. This model indicates that single species biofilm on porcine skin attains maturity (antibiotic tolerance) after 72 hours. The results of the mouse model demonstrate a 48-hour window for antibiotic susceptibility (and a 72-hour biocide susceptibility) as a planktonically-seeded surgical wound develops into a mature biofilm. However, our clinical results for wounds that were predominantly inoculated with $P$. aeruginosa suggest a $24-48$ hour window of increased antibiotic sensitivity for wound biofilm post-debridement.
This research was supported in part by grant number IP20GM078445-0I from the National Institute of General Medical Sciences (NIGMS), American Diabetes Association (grant \#, KR), Research and Testing intramural research funds. The contents of this report are solely the responsibility of the authors and do not necessarily represent the official views of the funding agencies 
References

I Cardinal, M., Eisenbud,

D.E., Armstrong, D.G. et al.

Serial surgical

debridement: a

retrospective study on

clinical outcomes in

chronic lower extremity

wounds. Wound Repair

Regen 2009; 17:3,

306-3II.

2 Falanga,V., Brem, H.,

Ennis,W.J. et al.

Maintenance debridement in the treatment of

difficult-to-heal chronic

wounds.

Recommendations of an expert panel. Ostomy

Wound Manage 2008; S2-

SI3.

3 Schultz, G.S., Sibbald,

R.G., Falanga, V. et al.

Wound bed preparation: a

systematic approach to

wound management.

Wound Repair Regen

2003; I I (Suppl. I), I-28.

4 Wolcott, R.D., Kennedy,

J.P., Dowd, S.E. Regular

debridement is the main

tool for maintaining a

healthy wound bed in

most chronic wounds.J

Wound Care 2009; 18: 2

54-56.

5 Medicare. TrailBlazer

Health Enterprises LCD

Titled Wound Care.

1/3/08.

Ref Type: Internet

Communication

6 James GA, Swogger E,

Wolcott R, et al. Biofilms

in chronic wounds. Wound

Repair Regen

2008:16(I):37-44.

7 Schaber, J.A., Triffo, W.
Suh, S.J. et al. Pseudomonas aeruginosa forms biofilms in acute infection

independent of cell-to-cell signaling. Infect Immun 2007; 75: 8, 37I5-372I.

8 Harrison-Balestra, C.,

Cazzaniga,A.L., Davis, S.C., Mertz, P.M.A woundsolated Pseudomonas aeruginosa grows a biofilm in vitro within 10 hours and is visualized by light microscopy. Dermatol Surg 2003; 29: 6, 63I-635. 9 Stewart PS, Costerton JW.Antibiotic resistance of bacteria in biofilms. Lancet 200।; 358: 9276, I35-138.

10 Stewart, P.S

Mechanisms of antibiotic resistance in bacterial biofilms. Int J Med Microbiol 2002; 292: 2 , 107-II3.

I I Russell AD.

Mechanisms of bacterial insusceptibility to biocides. Am J Infect Control 200I: 29: 4, 259-26I.

I 2 Stickler, D.J.

Susceptibility of antibiotic-resistant gram-negative bacteria to biocides: a perspective from the study of catheter biofilms. Symp Ser Soc Appl Microbiol 2002; 31 : 163S-170S.

13 Leid, J.G.,Willson, C.J., Shirtliff, M.E. et al. The exopolysaccharide alginate protects Pseudomonas aeruginosa biofilm bacteria from IFN-gamma mediated macrophage killing. J Immunol 2005 I75: II, 75।2-75 I8
I 4 Leid, J.G., Shirtliff, M.E., Costerton, J.W., Stoodley, A.P. Human leukocytes adhere to, penetrate, and respond to Staphylococcus aureus biofilms. Infect Immun 2002; 70: II,

6339-6345.

15 Costerton, J.W., Geesey, G.G., Cheng, K.J. How bacteria stick. Sci Am 1978; 238: I, 86-95.

16 Costerton, J.W.

Introduction to biofilm. Int Antimicrob Agents 1999; | : 3-4, 2|7-22|

I 7 Davies, D.G., Parsek,

M.R., Pearson, J.P. et al.The involvement of cell-to-cell signals in the development of a bacterial biofilm.

Science 1998; 280: 536I, 295-298.

18 Donlan, R.M

Costerton, J.W. Biofilms: survival mechanisms of clinically relevant microorganisms. Clin

Microbiol Rev 2002; 15: 2 , 167-193.

19 Hall-Stoodley, L.

Costerton, J.W., Stoodley, P. Bacterial biofilms: from the natural environment to infectious diseases. $\mathrm{Nat}$ Rev Microbiol 2004; 2: 2 , 95-108.

20 Stewart, P.S., Franklin, M.J. Physiologica

heterogeneity in biofilms.

Nat Rev Microbiol 2008; 6 : 3, 199-210.

2I Fux, C.A., Costerton,

J.W., Stewart, P.S., Stoodley, P. Survival strategies of infectious biofilms. Trends in Microbiology 2005; 13 : I, 34-40.
22 Allegrucci M, Hu FZ, Shen K, et al. Phenotypic characterization of

Streptococcus pneumonia biofilm development. J Bacteriol

2006; 188(7):2325-35.

23 Ehrlich GD, Hu FZ,

Shen K, Stoodley P, Post

JC. Bacterial plurality as

general mechanism driving persistence in chronic infections. Clin Orthop Relat Res 2005;(437):20-4

24 Ren, D., Bedzyk, L.A.

Thomas, S.M et al. Gene expression in Escherichic coli biofilms.Appl

Microbiol Biotechnol 2004; 64: 4, 515-524.

25 Cho, K.H., Caparon, M.G. Patterns of virulence gene expression differ between biofilm and tissue communities of

Streptococcus pyogenes. Mol Microbiol 2005; 57: 6, I545-1556.

26 Werner, E., Roe, F. Bugnicourt, A. et al. Stratified growth in Pseudomonas aeruginosa biofilms. Appl Environ Microbiol 2004; 70: 10, 6188-6196.

27 Rani, S.A., Pitts, B., Beyenal, $\mathrm{H}$. et al. Spatial patterns of DNA replication, protein synthesis, and oxygen concentration within bacterial biofilms reveal diverse physiological states. J Bacteriol 2007; 189. II , 4223-4233. 28 Sauer, K., Camper, A.K. Ehrlich, G.D et al.

Pseudomonas aeruginosa displays multiple

phenotypes during development as a biofilm. Bacteriol 2002; 184: II40. 29 Desrosiers, M., Myntti, M., James, G. Methods for removing bacterial biofilms: in vitro study using clinical chronic

rhinosinusitis specimens. Am J Rhinol 2007; 21: 5 , 527-532.

30 Schmook, F.P. Meingassner, J.G., Billich,A Comparison of human skin or epidermis models with human and animal skin in in vitro

percutaneous absorption. Int J Pharm 200।; 215: I-2 5I-56

3I Brown, R.L.

Greenhalgh, D.G. Mouse models to study wound closure and topical

treatment of infected wounds in healingimpaired and normal healing hosts. Wound Repair Regen 1997; 5: 2, 198-204.

32 Rumbaugh, K.P, Diggle, S.P., Watters, C.M. et al. Quorum sensing and the social evolution of bacterial virulence. Curr Biol 2009; | 9: 4, 34|-345 33 Holloway, B.W., Krishnapillai,V., Morgan, A.F. Chromosomal genetics of Pseudomonas. Microbiol Rev 1979; 43:।, 73-102.

34 Wolcott, R.D.

Gontcharova, V., Sun, Y.,

Dowd, S.E. Evaluation of

the bacterial diversity among and within individual venous le ulcers using bacterial tag-encoded FLX and titanium amplicon pyrosequencing and metagenomic approaches. BMC Microbiol 2009; 9 .

35 Rose, H, Baldwin, A., Dowson, C.G.

Mahenthiralingam, E. Biocide susceptibility of the Burkholderia cepacia complex. J Antimicrob Chemother 2009; 63: 3, 502-510.

36 Costerton, J.W., Stewart, P.S. Battling biofilms. Sci Am 200I; 285 I, 74-8I.

37 Stewart, P.S., Rayner, J., Roe, F., Rees, W.M. Biofilm penetration and disinfection efficacy of alkaline hypochlorite and chlorosulfamates. J Appl Microbiol 2001; 91 : 3, 525-532.

38 Stewart, P.S., Davison, W.M., Steenbergen, J.N. Daptomycin rapidly penetrates a Staphylococcus epidermidis biofilm. Antimicrob Agents

Chemother 2009; 53:8, 3505-3507.

39 Mah,T.F., O'Toole, G.A Mechanisms of biofilm resistance to antimicrobial agents. Trends Microbiol 2001; 9: 1, 34-39.

40 Stewart, P.S. Diffusion in biofilms. J Bacteriol 2003; |85: 5, |485-|49|.

\section{Journal of Wound Care}

